\title{
Nachhaltige Neuproduktentwicklung - ein interdisziplinärer Ansatz zur Identifikation von Rohstoffrisiken am Beispiel von Kobalt
}

\author{
Tobias Gaugler
}

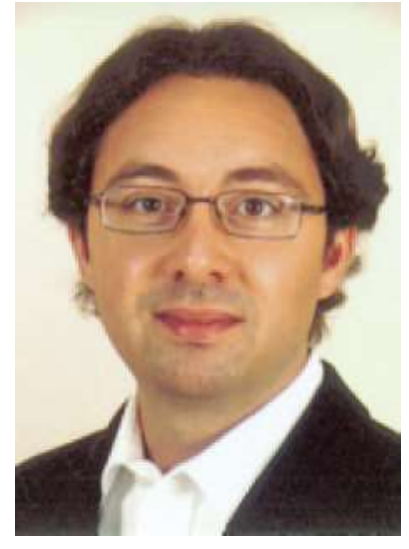

Tobias Gaugler

In einer Vielzahl entwickelter Volkswirtschaften (wie beispielsweiser der deutschen) ist das produzierende Gewerbe eine wichtige Stütze des materiellen Wohlstands. Um dieser Rolle - auch in Zukunft - gerecht werden zu können, ist der industrielle Sektor einerseits auf großvolumige Rohstoffimporte angewiesen. Um andererseits im Export global wettbewerbsfähig zu bleiben, stellt die Entwicklung innovativer Neuprodukte eine stetige Herausforderung dar. Da in der aktuellen wissenschaftlichen Literatur, die sich mit den Implikationen endlicher Rohstoffe auseinandersetzt, primär auf (techno-)ökonomische Aspekte fokussiert wird (EU 2010; Erdmann et al. 2011; Krohns et al. 2011), soll im Rahmen dieses Beitrags zudem auf ökologische und soziale Implikationen eingegangen werden. Nachdem zunächst mögliche Rohstoffrisiken diskutiert werden, die in jedem dieser Bereiche auftreten, werden mögliche Gegenmaßnahmen zu einer Nachhaltigkeits-Roadmap zusammengefasst.

\section{T. Gaugler $(\varangle)$}

Institut für Materials Resource Management (MRM), Universität

Augsburg, Universitätsstr. 2, 86159 Augsburg, Deutschland

E-Mail: tobias.gaugler@mrm.uni-augsburg.de
Um die Anwendbarkeit der vorgestellten Ansätze zu erleichtern, werden diese am Beispiel von Kobalt illustriert, das als strategisches Metall gilt (Angerer et al. 2009, S. 259). Der Beitrag zielt darauf ab, den Leser für die Notwendigkeit nachhaltige Neuproduktentwicklung zu sensibilisieren und dient als Diskussionsgrundlage fundierter unternehmerischer Entscheidungen im Bereich des nachhaltigen Ressourcenmanagements.

\section{Die Bedeutung von Rohstoffen für die Industrie}

Um die europäischen Union mittel- und langfristig wirtschaftlich zu stärken, kommt der Realwirtschaft eine Schlüsselrolle zu. Dies zeigen u. a. die Pläne der EU-Kommission, den Anteil der Industrie am BIP auf $20 \%$ im Jahr $2020 \mathrm{zu}$ steigern. Dieser im Vergleich zu heute $40 \%$ ige Anstieg soll durch eine Partnerschaft zwischen der EU, ihren Mitgliedsstaaten und der Industrie erreicht werden und Ausgangspunkt einer „,neuen industriellen Revolution“ (EU 2012, S. 4) sein. Das produzierende und verarbeitende Gewerbe kann - gerade in Zeiten globaler ökonomischer Verwerfungen - als Anker für Wohlstand angesehen werden, da es der mit einer Bruttowertschöpfung von über 600 Mrd. $€$ größte (Destatis 2012a) und aktuell stabilste deutsche Wirtschaftsbereich ist (Destatis 2012b, S. 9). Das produzierende bzw. verarbeitende Gewerbe wiederum ist in großem Umfang abhängig von einem sicheren Zugang zu Rohstoffen: Mit etwa 40 \% stellen Materialkosten den größten Anteil an dessen Gesamtkosten dar (Fraunhofer 2012); im Jahr 2010 mussten Rohstoffe im Wert von 109,3 Mrd. € nach Deutschland importiert werden (BGR 2011a, S. 19). Da bei Materialkosten im langfristigen Vergleich ein starker Preisanstieg erkennbar ist (Destatis 2007) und sich dieser Trend nach Auffassung der weit überwiegenden Zahl unternehmerischer Entscheidungsträger auch 
in Zukunft fortsetzen wird (Handelsblatt 2012), werden ein Anstieg der Rohstoffpreise sowie deren Schwankung aktuell als die beiden größten Gefahren für das Geschäftsergebnis sowohl von KMU als auch von großen Industrieunternehmen angesehen (Deutsche Bank 2012). Um ihre internationale Wettbewerbsfähigkeit halten bzw. ausbauen zu können, sind Unternehmen - insbesondere in den von Innovationen geprägten, wachstumsstarken Branchen wie Maschinenbau, Gesundheitstechnik, Automobil, Chemie, nachhaltige Mobilität, Umwelttechnologie und Ressourceneffizienz (Gönner 2010, S. 183) - darauf angewiesen, ihre Abnehmer mit qualitativ hochwertigen und technologisch führenden (Neu-)Produkten an sich zu binden.

Angerer et al. (2009) weisen darauf hin, dass insbesondere bei der Entwicklung und Produktion innovativer Neuprodukte mit einer verstärkten Nachfrage nach Rohstoffen zu rechnen ist. Dieser Anstieg ist einerseits quantitativer Natur. Andererseits erhöhte sich auch die Anzahl der nachgefragten Elemente, deren spezifische Materialeigenschaften für eine Vielzahl von Zukunftstechnologien essentiell sind, von ca. 25 in den 1970er Jahren auf über 70 (Achzet et al. 2011, S. 11; Krohns et al. 2011, S. 899). Industrieunternehmen, die wie beschrieben in besonderem Maße von der Versorgung mit Rohstoffen angewiesen sind, stehen somit insbesondere bei der Entwicklung neuer Produkte vor einer doppelten Herausforderung: Einerseits ist bereits in der Produktentwicklung bzw. im Produktdesign darauf zu achten, die zum Einsatz vorgesehenen Rohstoffe auf ihre Kritikalität hin zu überprüfen. Es gilt, den Einsatz von als kritisch identifizierten Rohstoffen zu reduzieren bzw. diese durch weniger kritische Rohstoffe zu substituieren (Buhl et al. 2010). Andererseits muss ein Unternehmen sicherstellen, dass die für die Produktion benötigten Rohstoffe über den gesamten Lebenszyklus des Produkts bzw. der Produktreihe sowohl in Bezug auf die Menge als auch in Bezug auf den kalkulierten Einkaufspreis zur Verfügung stehen. Zudem kommt einem Unternehmen eine soziale sowie ökologische Verantwortung zu, die sich zunehmend auch auf den Bezug von Rohstoffen bezieht (BMU 2012).

\section{Praxisbeispiel: Die Rolle von Kobalt für leistungsfähige Energiespeicher}

Zur Verdeutlichung des im Rahmen dieses Beitrags vorgestellten Nachhaltigkeits-Roadmap soll beispielhaft auf ein Unternehmen eingegangen werden, das in der Entwicklung und Herstellung von wiederaufladbaren, elektrischen Energiespeichern tätig ist, die nicht zuletzt im Zuge der Energiewende von zunehmender Bedeutung sind (DLR 2012). Abhängig von den Anforderungen an die Leistungsbzw. Energiedichte eines solchen Akkumulators stehen hierzu grundsätzlich mehrere Speichertechnologien zur Verfügung wie beispielsweise Elektrolytkondensatoren,
Doppelschichtkondensatoren sowie Batterien auf Blei-, Nickel-Cadmium-, Nickel-Metallhydrid-, Zink-Luft- bzw. Lithium-Ionen-Basis (Braess und Seiffert 2005, S. 116). Aus technischer Sicht zeichnen sich Lithium-Ionen-Akkumulatoren durch die aktuell höchste Energiedichte aus und werden als ,die Stromspeichertechnologie der Zukunft“ angesehen (Angerer et al. 2009, S. 171 und S. 260). Nachdem davon ausgegangen werden kann, dass die langfristige Versorgung mit Lithium gewährleistet ist (Yaksic und Tilton 2009), stellt Kobalt die Engpassressource dieser Technologie dar (Angerer et al. 2009, S. 173). Vor diesem Hintergrund soll dieses Metall im vorliegenden Artikel als Anwendungsbeispiel zum nachhaltigen Ressourcenmanagement dienen.

\section{Rohstoff-Basisinformationen}

Bevor in den folgenden Abschnitten genauer auf die ökonomischen, ökologischen und sozialen Auswirkungen des Ressourcenbedarfs eingegangen werden kann, die sich für ein produzierendes Unternehmen aus der Entwicklung eines Neuproduktes ergeben, muss zunächst ein Mengengerüst aufgestellt werden. Hierbei gilt es, die folgenden Fragen zu beantworten:

- Welche Rohstoffe werden für die Produktion des Neuproduktes benötigt?

- Wie viele Endprodukte sollen pro Jahr abgesetzt werden? In welcher Menge wird jeder dieser Rohstoffe für die Herstellung eines Endprodukts benötigt?

- Von welchem Produktlebenszyklus wird ausgegangen?

- Ergeben sich aus der Festlegung auf bestimmte Rohstoffe Implikationen auf weitere Produkte bzw. Produktreihen?

Eine exemplarische Aufstellung des Kobalt-Mengengerüsts für die a.o.S. bereits eingeführte Herstellung von Energiespeichern auf Lithium-Ionen-Basis ergibt für ein Unternehmen, das einen Weltmarktanteil von $4 \%$ anstrebt, einen geschätzten Bedarf von 650 t Kobalt im Jahr 2012, der sich bedingt durch die steigende Nachfrage auf ca. $900 \mathrm{t}$ im Jahr 2020 erhöhen und zum unterstellten Ende der Produktreihe im Jahr 2030 ca. 1.200 t betragen wird (Durchschnittsszenario nach Angerer et al. 2009, S. 173). Für den gesamten Planungszeitraum entspricht dies einem Gesamtbedarf von ca. 17.000 t Kobalt.

Um abschätzen zu können, inwieweit sich aus dem Bedarf nach einzelnen Rohstoffen Risiken für eine nachhaltige Neuproduktentwicklung ergeben, ist zudem ein Überblick über die Abbauländer nötig. Wird diese Untersuchung wiederum am Beispiel von Kobalt durchgeführt, wird deutlich, dass der Rohstoffabbau primär in Ländern stattfindet, die bzgl. ihrer wirtschaftlichen bzw. politischen Stabilität als kritisch einzuschätzen sind. So stam- 
Abb. 1 Weltweiter Kobaltabbau nach Ländern (in \%) sowie deren politische Stabilität (Skala: 0-100 Punkte). (Eigene Darstellung mit Daten von USGS 2010 und Weltbank 2010)

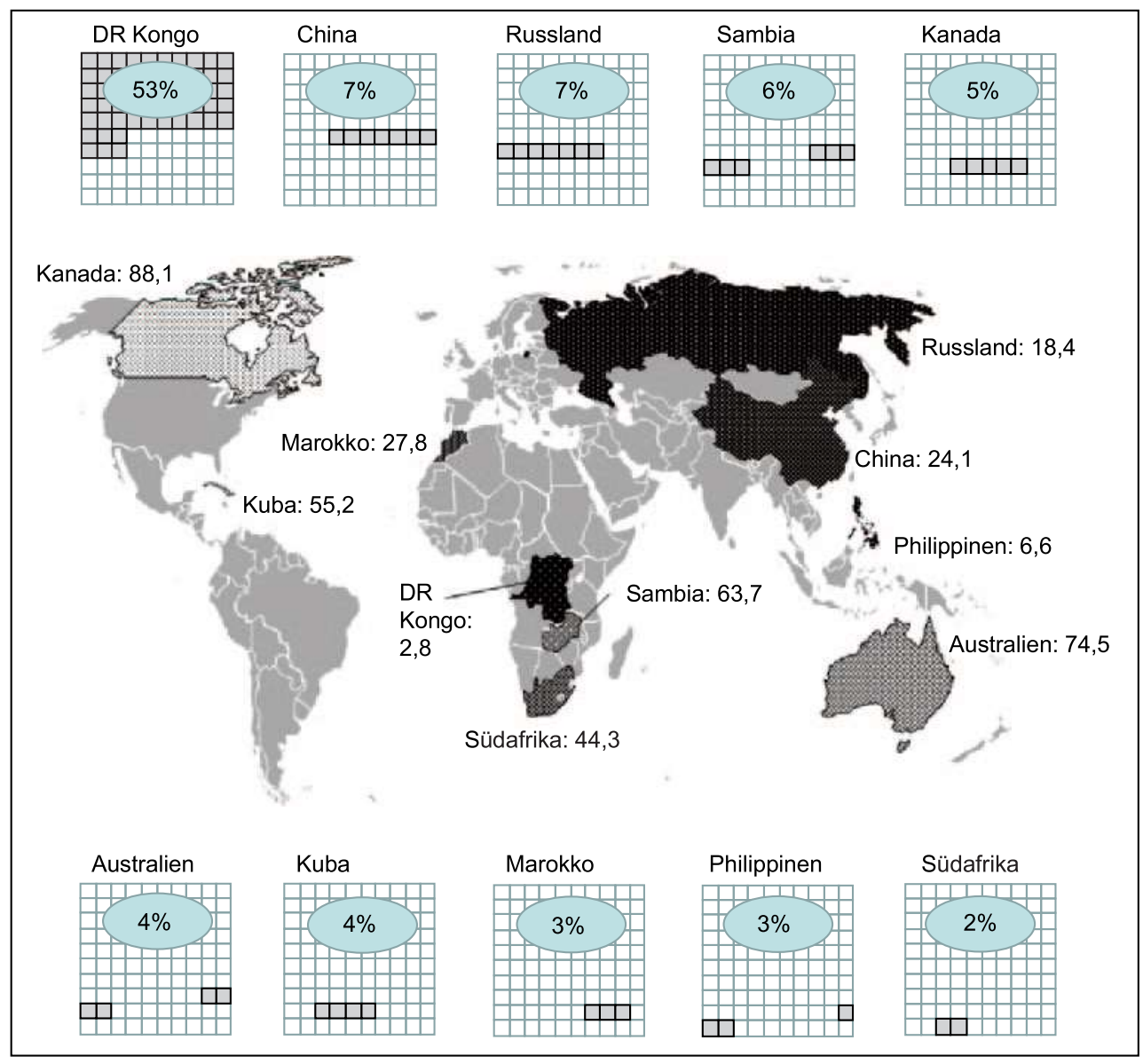

men $53 \%$ der weltweiten Minenproduktion aus der DR Kongo, jeweils $7 \%$ aus China und Russland sowie $6 \%$ aus Sambia (USGS 2010). Weitere Informationen über den weltweiten Kobaltabbau (Top 10 Abbauländer) sowie die politische Stabilität der Abbauländer sind in Abb. 1 zusammengestellt.

\section{3 Ökonomische Aspekte}

Im Folgenden wird auf die ökonomischen Implikationen eingegangen, die sich aus dem Rohstoffbedarf eines Unternehmens ergeben. Hierbei werden zunächst - wiederum anhand konkreter Fragestellungen - mögliche Risiken und sowie deren Auswirkungen vorgestellt. Darauf aufbauend wird im zweiten Teil des Abschnitts auf konkrete Maßnahmen eingegangen, die ein Unternehmen zur Reduktion dieser Risiken einsetzten kann.

Ökonomische Risiken beziehen sich in den meisten Fällen direkt auf den Preis, zu dem ein Rohstoff dem Unternehmen aktuell und zukünftig zur Verfügung steht. Hierzu bedarf es der wiederum rohstoffspezifischen Beantwortung der folgenden Fragen:
- Was kostet der Rohstoff heute?

- Wie hoch sind die rohstoffspezifischen Kosten, die für das Unternehmen anfallen?

- Welche ökonomische Kritikalität wird dem Rohstoff für die Zukunft zugemessen?

- In welche Richtung wird sich der Preis des Rohstoffs zukünftig entwickeln?

- Mit welchen Preisschwankungen ist zu rechnen?

- Bestehen (kurzfristige) Verfügbarkeitsrisiken?

Im Fall des Kobalt-verarbeitenden Unternehmens ergeben sich bei einem aktuellen Preis von ca. 37.000 \$/t (Metalprices 2012) rohstoffspezifische Kosten von ca. 24 Mio. \$ im Jahr 2012. Unter der (unrealistischen) Annahme unveränderter Rohstoffpreise und dem a.o.S. dargestellten Nachfrageanstieg ergeben sich bis zum Jahr 2030 aufsummierte Kosten für den Kauf von Kobalt i.H.v. ca. 629 Mio. \$. Um die künftige Preisentwicklung abschätzen zu können, lassen sich rohstoffspezifische Kritikalitätsindikatoren heranziehen, die beispielsweise die (statische bzw. dynamische) geologische Verfügbarkeit, Mengenrelevanz, Länder- und Unternehmenskonzentrationen, politische Rahmenbedingungen und andere Risiken zu einem Indikator verdichten (vgl. Graedel et al. 2011). Im Fall von Kobalt gehen Erd- 


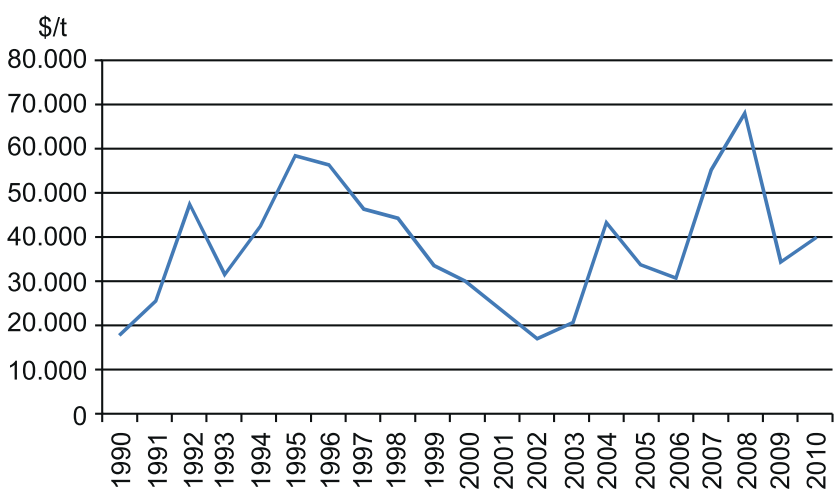

Abb. 2 Historischer Kobaltpreis 1990-2010. (Eigene Darstellung mit Daten von USGS 2012)

mann et al. (2011, S. 44) von einer mittleren Kritikalität aus. Demgegenüber zählt Kobalt zu den „,14 critical raw materials at EU level“". Auch wenn Prognosen über die Preisentwicklung von Rohstoffen grundsätzlich mit Vorsicht zu begegnen ist und seitens der Wissenschaft Uneinigkeit darüber besteht, wie sich Rohstoffpreise entwickeln (Slade 1982; Svedberg und Tilton 2006; Radetzki 2008), ist auf Basis der o.g. Studien davon auszugehen, dass die mittlere bzw. hohe Kritikalität sowie die zunehmende Nachfrage nach Kobalt (BGR 2011a, S. 137) zu tendenziell steigenden Preisen führen werden. Wird hierbei ein jährlicher Preisanstieg von $3 \%$ angenommen, belaufen sich die Mehrkosten für den Kauf von Kobalt im Beispiel auf ca. 200 Mio. \$. In Bezug auf die zu erwartenden Volatilität von Rohstoffpreisen muss davon auszugehen werden, dass diese in Zukunft weiter ansteigen wird (Goldman Sachs 2011, S. 5). Zudem legt die Untersuchung des historischen Preisverlaufs (s. Abb. 2) nahe, dass auch mit zukünftig starken Preisschwankungen gerechnet werden muss.

Da davon ausgegangen werden kann, dass Kobalt-Lieferungen aus der rohstoffexportabhängigen DR Kongo (Knoke und Binnewies 2010, S. 7), auch zukünftig erfolgen werden, zudem in den nächsten Jahren weiterer KobaltMinen auch in politisch stabilen Ländern ihre Produktion aufnehmen werden (USGS 2010) und die (statische) Reichweite aktuell bei ca. 110 Jahren liegt (Frondel et al. 2007, S. 13), ist damit zu rechnen, dass eine unterbrechungsfreie Verfügbarkeit von Kobalt sichergestellt ist.

Auf Basis der ökonomischen Risikoeinschätzung, die für jeden Rohstoff separat zu erfolgen hat, bestehen nun verschiedene Möglichkeiten, diese Risiken zu reduzieren. Diese umfassen (nach aufsteigendem Aufwand)

- die Absicherung der Rohstoffkosten durch Lagerhaltung oder Termingeschäfte (Hedging) (Deutsche Bank 2007, S. 5), wobei diese Strategie zumeist nur mit kurzen bzw. mittelfristigen Laufzeiten und lediglich für wenige Rohstoffe angeboten wird,
- die Abwälzung des Rohstoffpreisrisikos auf die Kunden beispielsweise durch Preisanpassungen oder die vorherige Vereinbarung von Preisgleitklauseln,

- den Aufbau von langfristigen Lieferverträge mit Rohstoffproduzenten und damit verbundenen, langfristigen Preisvereinbarungen,

- die Suche nach neuen Lieferanten, die ihre Rohstoffe aus unterschiedlichen Förderländern beziehen und somit zu einer Diversifikation des Länderrisikos beitragen,

- die Steigerung der eigenen F\&E-Aktivitäten mit dem Ziel, die Ressourceneffizienz zu steigern und somit die Rohstoffkosten zu senken,

- die Substitution von Rohstoffen hoher Kritikalität durch mittel- und langfristig sichererer Alternativrohstoffe, sofern technische Eigenschaften und Restriktionen dies zulassen,

- den Auf- oder Ausbau eines unternehmensinternen Ressourcenmanagement-Systems, in dem sich Daten über den aktuellen und zukünftigen Roststoffverbrauch mit den o.g. Preis- und Verfügbarkeitsrisiken verknüpfen lassen (IHK 2010, S. 4) und eine gezielte Rohstoffplanung bereits in der Phase der Neuproduktentwicklung erleichtern,

- den Bezug von Sekundärrohstoffen bzw. den Aufbau eines eigenen Recyclingsystems z. B. in Verbindung mit der Einführung eines Pfandes bzw. einer Rückgabeprämie auf Altprodukte sowie

- die eigene Erschließung von Rohstoffquellen (vertikale Rückwärtsintegration) bzw. die Zusammenarbeit mit Organisationen (wie bspw. der Deutschen Rohstoffagentur), die die Exploration mittel- und langfristig sicherer Rohstoffquellen forcieren (Buhl et al. 2010; Achzet et al. 2011; DERA 2012).

Inwieweit die hier vorgestellten ökonomischen Maßnahmen auf den Rohstoff Kobalt anwendbar sind, lässt sich der folgenden Aufstellung sowie - im Detail - den ebenfalls angeführten Quellen entnehmen (Tab. 1).

\section{4 Ökologische Aspekte}

Nach der Diskussion ökonomischer Risiken sowie möglicher Strategien, diese zu reduzieren, soll nun auf ökologische Treiber eingegangen werden, die im Rahmen einer nachhaltigen Neuproduktentwicklung und -produktion Berücksichtigung finden müssen. Beweggründe, auch ökologische Risiken näher zu betrachten, können sich einerseits aus einer unternehmerischen Verantwortung gegenüber der Umwelt herleiten, wie dies bspw. auch seitens der EU gefordert wird (EU 2011, Abs. 65) - selbst wenn diese u. U. mit rein ökonomischen Interessen kon- 
Tab. 1 Einzelmaßnahmen zur Reduktion ökonomischer Rohstoffrisiken

\begin{tabular}{|c|c|c|}
\hline Mögliche Einzelmaßnahmen & Fallbeispiel Kobalt & Quelle \\
\hline Lagerhaltung & $\begin{array}{l}\text { In firmeneigenen Lagern oder bspw. in Lagern der } \\
\text { LME (aktueller Preis: } 0,53 \$ / t / T a g \text { ) möglich }\end{array}$ & LME (2012a) \\
\hline Abschluss von Termingeschäften & $\begin{array}{l}\text { Absicherung über Kobalt-Futures über die LME } \\
\text { möglich (seit 2010) }\end{array}$ & LME (2010); LME (2012b) \\
\hline Abwälzung des Rohstoffpreisrisikos & $\begin{array}{l}\text { Abhängig von Preissensitivität der Kunden und } \\
\text { Konkurrenzsituation möglich }\end{array}$ & Berger (2012, S. 5 ff.) \\
\hline $\begin{array}{l}\text { Lieferantenauswahl und Abschluss } \\
\text { langfristiger Lieferverträge }\end{array}$ & Abhängig vom Mengenbedarf möglich & Cobalt Development Institute (2012, S. 3) \\
\hline Steigerung der Ressourceneffizienz & Möglich, jedoch abhängig von F\&E-Intensität & Ellis et al. (2007) \\
\hline Substitution & Nur eingeschränkt möglich & BGR (2007, S. 8); Angerer et al. (2009, S. 173) \\
\hline $\begin{array}{l}\text { Aufbau eines } \\
\text { Ressourcenmanagement-Systems }\end{array}$ & $\begin{array}{l}\text { Je nach Abhängigkeit von kritischen Rohstoffen } \\
\text { skaliert möglich }\end{array}$ & Buhl et al. (2010); Clausmark (2012) \\
\hline $\begin{array}{l}\text { Einsatz von Sekundärrohstoffen/ } \\
\text { Recycling }\end{array}$ & $\begin{array}{l}\text { Technisch möglich; ökonomisch Einsatz jedoch } \\
\text { abhängig vom Preis des Primärrohstoffs }\end{array}$ & $\begin{array}{l}\text { Faulstich et al. (2010, S. 31); Treffer (2011, } \\
\text { S. 67); USGS (2012, S. 14) }\end{array}$ \\
\hline Vertikale Rückwärtsintegration & Abhängig vom Mengenbedarf möglich & RA (2012) \\
\hline
\end{tabular}

fligiert. Andererseits können Unternehmen der Erreichung bzw. bewussten Übererreichung ökologische Ziele auch einen ökonomischen Stellenwert zumessen, wenn sich dadurch ein positives Unternehmensimage aufbauen lässt. Durch nachhaltige Unternehmensführung sinkt zudem das Risiko, dass negative mediale Berichterstattung über mangelnde Umweltstandards oder eine auf mangelnde Umweltstandards zurückzuführende Umweltkatastrophe die Reputation des Unternehmens direkt oder indirekt gefährdet.

Umweltauswirkungen, die sich aus dem Bedarf nach Rohstoffen ergeben, treten während der Ausgrabung und Förderung des Erzes, der Erzanreicherung sowie bei der Verhüttung (Oertel 2003, S. 2), aber auch in nachgelagerten Phasen auf und stellen die folgenden Fragen in den Mittelpunkt:

- Ist mit morphologischen Auswirkungen wie beispielsweise Senkungstendenzen oder der Entstehung von Abraumhalden zu rechnen?

- Bewirkt der Rohstoffabbau Störungen des Wasserhaushaltes (hydrologische Auswirkungen)?

- In welchem Maße werden Flora und Fauna vor Ort in Mitleidenschaft gezogen?

- Mit welchen atmosphärischen Begleiterscheinungen muss gerechnet werden (Reller und Meißner 2012, S. 15)?

- Welche Auswirkungen auf die Umwelt fallen durch den Transport des Rohstoffs an?

- Entstehen am Ende der Nutzungsphase Gefahren aus der Entsorgung des Rohstoffs?

Werden diese Fragestellungen in Bezug auf den Abbau von Kobalt angewandt, wird deutlich, dass morphologische Auswirkungen aktuell geringe Relevanz haben. Dies ist der Tatsache geschuldet, dass der Kobalt-Abbau überwiegende artisanal betriebene wird und der Einsatz groß- technischen Geräts somit meist unterbleibt (Tsurukawa et al. 2011, S. 19). Demgegenüber stellen die aus dem Kobaltabbau resultierenden Abwässer sowie der Abraum eine große Umweltgefährdung dar, da die darin enthaltenen Giftstoffe wie Arsen, Kadmium und Blei in Entwicklungsländern zumeist ungeklärt bleiben (Heydenreich und Schelhove 2012). Zur Produktion von $1 \mathrm{~kg}$ Kobalt werden ca. 125 MJ-Äquivalente Energie und ca. 36.0001 Wasser benötigt (EcoInvent 2010a). Zudem wird die Luft durch die Produktion von $1 \mathrm{~kg}$ Kobalt mit $8,3 \mathrm{~kg} \mathrm{CO}$-Äquivalenten verschmutzt (EcoInvent 2010b). Für die Zukunft ist zu befürchten, dass die aus dem Kobaltabbau resultierenden Umweltbelastungen weiter zunehmen: Einerseits erzwingen die in den meisten Kobalt fördernden Ländern herrschenden wirtschaftlichen Rahmenbedingungen die Minenarbeiter, sich über die (zumindest teilweise vorhandenen) Umweltauflagen hinwegzusetzen. Zum anderen ist festzustellen, dass staatseigene chinesischen Rohstoffunternehmen, die seit 2008 in der DR Kongo knapp 10 \$ Mrd. $\$$ in den Ressourcenabbau und die hierfür nötige Infrastruktur investieren, ebenso wie indische Abbauunternehmen und Rohstoffhandelshäuser einem umweltverträglichen Kobaltabbau eine sehr geringe Bedeutung zumessen (Tsurukawa et al. 2011, S. 17 ff.). Demgegenüber spielten die Umweltauswirkungen, die sich aus dem Transport der aktuell p.a. geförderten knapp 100.000 t Kobalt ergeben (USGS 2012, S. 47), lediglich eine untergeordnete Rolle. Bei ordnungsgemäßer Entsorgung der Lithium-Ionen-Batterie am Ende der Nutzungsphase geht vom hier untersuchten Kobalt keine Gefährdung für die Umwelt aus (Treffer 2011, S. 66).

Nach der Diskussion der aus dem Rohstoffabbau resultierenden Umweltrisiken sollen nun Maßnahmen vorgestellt werden, die aus ökologischer Perspektive zu einer nachhaltigeren Rohstoffnutzung beitragen können. Auch 
hierbei gilt es, die ökologischen Risiken bezogen auf jeden Rohstoff einzeln zu untersuchen und Gegenmaßnahmen insbesondere bei denjenigen Rohstoffen zu forcieren, deren Abbau - abhängig von der seitens des Unternehmens benötigten Rohstoffmenge - die größten Umweltschädigungen nach sich ziehen. Die folgenden Einzelmaßnahmen können unterschieden werden:

- die Auswahl von Rohstofflieferanten, die umweltverträglichen Rohstoffabbau unter Einhaltung ökologischer Mindeststandards nachweisen können; beispielsweise durch Zertifikate anerkannter Träger und dem (verstärkten oder ausschließlichen) Einkauf von Rohstoffen aus Ländern, die über eine Umweltgesetzgebung mit hohen Standards verfügen sowie für deren Einhaltung Sorge tragen,

- die Reduktion des Bedarfs an umweltkritischen Rohstoffen durch (bereits im vorhergehenden Abschnitt vorgestellte Maßnahmen wie) die Steigerung der Ressourceneffizienz, Substitution durch umweltverträglichere Rohstoffe und den Bezug von Sekundärrohstoffen aus Recyclingsystemen,

- die Förderung der Erforschung von Technologien, die es ermöglichen, die geographische Herkunft von Rohstoffen beispielsweise anhand chemisch-mineralogischer Fingerprints nachzuweisen und so Handelsketten besser rückverfolgen zu können (BGR 2010),

- eine ideelle bzw. finanzielle Unterstützung von Initiativen und Organisationen, die sich für umweltgerechten Rohstoffabbau bzw. die Beseitigung von belasteten Abwässer und Abraum einsetzen sowie

- die aktive Kommunikation des ökologischen Engagements, um aktuelle bzw. potenziellen Kunden sowie die Öffentlichkeit über dieses Alleinstellungsmerkmal zu informieren.

Die Anwendbarkeit dieser Maßnahmen ist abhängig von der unternehmensspezifischen Rohstoffabhängigkeit und soll hier wiederum beispielhaft für Kobalt dargestellt werden (Tab. 2).

\section{Soziale Aspekte}

Um zudem auf einen fairen und partnerschaftlichen Umgang mit den Menschen hinarbeiten zu können, die im Abbau und der Aufbereitung von Rohstoffe tätig sind, stehen soziale Auswirkungen des Rohstoffabbaus im Mittelpunkt des folgenden Abschnitts. Diese betreffen zunächst die direkt im Bergbau Beschäftigten, indirekt jedoch auch die Gemeinden in den Abbauregionen sowie die Gesellschaft des Abbaulandes als Ganzes (Benoît und Mazijn 2009, S. 26). Die Umsetzung dieser Maßnahmen kann ökonomisch motiviert sein, um bspw. Streiks und damit verbundenen Lieferausfälle oder Reputationsrisiken zu vermeiden. Die Übererfüllung sozialer Standards lässt sich jedoch auch aus einem Verantwortungsgefühl den dortigen Beschäftigten gegenüber rechtfertigen. Hierbei stehen die folgenden Fragestellungen im Mittelpunkt:

- Treten aufgrund der a.o.S. diskutierten Umweltschädigungen ernste gesundheitliche Beeinträchtigungen der Bewohner von Abbaugebieten auf? Kommt es zu qualitativen bzw. quantitativen Ernteeinbußen, die auf eine abbaubedingte Schadstoffbelastung der Böden zurückzuführen sind? In welchem Maße werden Luft und Wasser in Mitleidenschaft gezogen?

- Welche Arbeitsbedingungen herrschen in den Abbauregionen vor? Werden die Beschäftigen angemessen entlohnt, gelten zumutbare Arbeitszeiten sowie Vorschriften zur Arbeitssicherheit? Haben die Arbeitnehmer die Möglichkeit, sich bspw. gewerkschaftlich zu organisieren bzw. Arbeitnehmerrechte durchzusetzen? Findet Kinderarbeit statt?

- Welche Rolle spielt der Staat für eine gerechte Verteilung des aus dem Rohstoffabbau resultierenden Reichtums? Bestehen soziale Sicherungssysteme? Welche Rolle hat Korruption in dem Rohstoff abbauenden Land?

- Bestehen soziale Spannungen oder kriegerische Auseinandersetzungen, die durch Gelder aus dem Rohstoffschmuggel befeuert werden? Führten Kämpfe um

Tab. 2 Einzelmaßnahmen zur Reduktion ökologischer Rohstoffrisiken

\begin{tabular}{|c|c|c|}
\hline Mögliche Einzelmaßnahmen & Fallbeispiel Kobalt & Quelle \\
\hline $\begin{array}{l}\text { Auswahl ökologisch verantwortlicher } \\
\text { Rohstofflieferanten }\end{array}$ & Abhängig vom Mengenbedarf möglich & $\begin{array}{l}\text { BGR (2011b); Cobalt Development } \\
\text { Institute (2012, S. 3); EPI (2012) }\end{array}$ \\
\hline $\begin{array}{l}\text { Reduktion des Bedarfs an ökologisch kritischen } \\
\text { Rohstoffen }\end{array}$ & $\begin{array}{l}\text { Möglich, sofern dies auch techno-ökonomisch } \\
\text { möglich ist }\end{array}$ & $\begin{array}{l}\text { Ellis et al. (2007); BGR (2007, S. 8); } \\
\text { Angerer et al. (2009, S. 173) }\end{array}$ \\
\hline $\begin{array}{l}\text { Förderung von Technologien zur Ermittlung der geo- } \\
\text { graphische Herkunft von Rohstoffen }\end{array}$ & $\begin{array}{l}\text { In Analogie zum sog. „Coltan-Fingerprint“" } \\
\text { möglich }\end{array}$ & BGR (2010) \\
\hline $\begin{array}{l}\text { Unterstützung von Initiativen und Organisationen, } \\
\text { die sich für umweltverträglichen Bergbau einsetzen }\end{array}$ & $\begin{array}{l}\text { Möglich; beispielsweise durch die Unter- } \\
\text { zeichnung des von deutschen Nachhaltig- } \\
\text { keitsrat initiierten „Kodex für Nachhaltigkeit““ }\end{array}$ & Nachhaltigkeitsrat (2012) \\
\hline $\begin{array}{l}\text { Aktive Kommunikation des ökologischen } \\
\text { Engagements }\end{array}$ & Stets möglich & - \\
\hline
\end{tabular}


rohstoffreiche Gebiete zu gewalttätigen Auseinandersetzungen, Bürgerkriegen und damit verbundenen Flüchtlingsströmen?

In Bezug auf den Abbau von Kobalt hat die soziale Komponente der Nachhaltigkeit einen hohen Stellenwert, da der weit überwiegende Teil in artisanalem Bergbau gewonnen wird. In den letzten 10 Jahren wurden beispielsweise in der Region Katanga/Kongo, in der die Hälfte des globalen Kobaltabbaus stattfindet, 60-90\% des Kobalts im manuellen Kleinbergbau abgebaut. Allein dort sind ca. 75.000 Menschen Vollzeit im Kobaltabbau tätig; in Zeiten großer Nachfrage steigt ihre Zahl auf über 100.000 Minenarbeiter an (Tsurukawa et al. 2011, S. 5 ff.). Das größte gesundheitliche Problem ist die Radioaktivität des Erzes, aus dem Kobalt gewonnen wird (US Embassy 2010). Dies führt u. a. dazu, dass Bewohner, die in einem Radius von $10 \mathrm{~km}$ um ein Gebiet mit Minenaktivität lebt, extrem erhöhte Uran-, aber auch Kobalt-, Blei- und Cadmium-Belastung aufwiesen (Tsurukawa et al. 2011, S. 39). Zudem vernichtet die radioaktive Verseuchung der Böden die Einkommensquelle der Bauern in den Regionen, in denen Kobalt abgebaut wird (Heydenreich und Schelhove 2012). Noch größere direkte Folgen hat die radioaktive Strahlung für die Bergarbeiter, die direkt mit dem Kobalterz in Kontakt sind. In einzelnen Minen sind Arbeiter Strahlungsdosen von bis zu $24 \mathrm{mSv} / \mathrm{J}$ ahr ausgesetzt (während bspw. in der EU für strahlenexponierte Volljährige ein Grenzwert von $20 \mathrm{mSv} /$ Jahr gilt). Der Verdienst katangischer Bergleute beläuft sich auf ca. 3-5 \$ pro Tag, ist damit verglichen mit dem Durchschnittseinkommen einer fünfköpfigen Familie (ca. 2,5\$) relativ hoch und kompensiert insoweit auch die hohe Zahl an Überstunden. Da Sicherheitsvorschriften im artisanalen Bergbau oftmals nicht eingehalten werden, liegt die Rate der pro Jahr tödlich verunglückten Bergarbeiter - je nach Mine - zwischen 0,1 und 0,5\%. Da meist nur zeitlich befristete Arbeitsverträge gelten und nur 0,5\% der Minenarbeiter gewerkschaftlich organisiert sind, können Arbeitnehmerrecht nur schwerlich durgesetzt werden. Zudem ist Kinderarbeit weit verbreitet: $28 \%$ der Beschäftigten im Kobaltabbau sind unter 15 Jahre alt; weitere $14 \%$ sind 15 - bis 17-Jährige. $73 \%$ der Kongolesen gelten als arm (Tsurukawa et al. 2011, S. 28 ff.) und soziale Sicherungssysteme sind nur rudimentär ausgeprägt. Dies liegt u. a. daran, dass der Regierung der DR Kongo - mangels transparenter Handelsketten - bis zu 90 \% der möglichen Steuereinnahmen aus dem Rohstoffexport verloren gehen (Nachhaltigkeitsrat 2010). Zudem belegt die DR Kongo in Bezug auf Korruption weltweit Platz 168 von 182 (Transparency International 2011). Ethische Spannungen haben in den letzten Jahren an Intensität verloren; zuletzt waren im Jahr 1992 bei Auseinandersetzungen unterschiedlicher Volksgruppen 3.000 Tote und 500.000 Vertriebene zu beklagen (Tuseko 2001).
Abhängig davon, welche Rohstoffe in welcher Menge bei dem zu entwickelnden Neuprodukt benötigt werden und der Intensität der rohstoffspezifischen Gefährdung empfiehlt es sich für ein nachhaltig orientiertes Unternehmen, geeignete Maßnahmen zu ergreifen. Hierzu bietet es sich insbes. an,

- das gesamte Zulieferernetzwerk zu verpflichten, soziale (Mindest-)Standards einzuhalten (i. V. m. der Vereinbarung von Strafen im Falle der Nichteinhaltung sowie Kontrolle der Umsetzung) und im Gegenzug langfristige Lieferverträge mit kooperierenden Zulieferunternehmen abzuschließen, die wiederum deren Planungs- und Investitionssicherheit erhöht,

- sich gegenüber Vorlieferanten, Unternehmerverbänden sowie staatlichen Stellen für Transparenz in Bezug auf die Rohstoffhandelsströme einzusetzen und sich hierbei sowohl auf Waren- als auch auf Finanzströme zu beziehen,

- Initiativen und Organisationen zu unterstützen, die Schulungsmaßnahmen in den Rohstoffabbauregionen unterstützen, in denen Minenarbeitern in Bezug auf gesundheitliche Risiken sowie mögliche Schutzmaßnahmen aufklärt werden,

- den Bedarfs nach Rohstoffen zu reduzieren, die unter menschenunwürdigen Arbeitsbedingungen produziert bzw. um die Rohstoffkriege geführt werden (bspw. durch Steigerung der Ressourceneffizienz, Substitution, Bezug von Sekundärrohstoffen) sowie

- Maßnahmen, die das Unternehmen in Bezug auf den fairen Umgang mit Mitarbeitern auch vorgelagerter Produktionsschritte umsetzt, zum Aufbau eines positiven Unternehmensimages zu nutzen. So wäre es - insbes. aus der Sicht der betroffenen Arbeiter - wünschenswert, dass hierdurch im Rohstoffbereich ein ähnliches gesellschaftliches Umdenken beginnt wie dies vor Jahrzehnten bei Lebensmitteln wie Kaffee oder Kakao der Fall war (Hütz-Adams 2012, S. 4).

Die Verbesserung der Lebensbedingungen in den heute oftmals von Armut und Kriegen betroffenen rohstoffexportierenden Ländern stellt eine langwierige und komplexe Herausforderung für eine Vielzahl von Akteuren dar. Vor diesem Hintergrund bietet es sich für ein nachhaltig agierendes Unternehmen an, in Zusammenarbeit mit seinen Vorlieferanten und NGOs zudem eine langfristige Roadmap zur Einhaltung bzw. Steigerung von Sozialstandards in Abbauländern $\mathrm{zu}$ entwickeln und umzusetzen. Mittels dieser können die o.g. Einzelmaßnahmen stufenweise eingeführt sowie deren Standards sukzessive erhöht werden.

Auch für den sozialen Aspekt nachhaltiger Neuproduktentwicklung wird - wiederum am Beispiel von Kobalt und mit Verweis auf weitere Quellen - auf die Umsetzbarkeit der vorgestellten Maßnahmen eingegangen (Tab. 3). 
Tab. 3 Einzelmaßnahmen zur Reduktion sozialer Rohstoffrisiken

\begin{tabular}{|c|c|c|}
\hline Mögliche Einzelmaßnahmen & Fallbeispiel Kobalt & Quelle \\
\hline $\begin{array}{l}\text { Definition sozialer (Mindest-) Anforderungen/ } \\
\text { Zertifizierung und Durchsetzung gegenüber allen } \\
\text { Zulieferern }\end{array}$ & $\begin{array}{l}\text { Möglich unter Einhaltung ökonomischer Neben- } \\
\text { bedingungen; Zertifizierung bspw. in Analogie zum } \\
\text { Zinnabbau in der DR Kongo (BGR 2011b) }\end{array}$ & BMZ (2010, S. 16); ILO (2012) \\
\hline $\begin{array}{l}\text { Forcierung der Transparenz in Bezug auf die } \\
\text { Rohstoff- sowie deren Finanzströme }\end{array}$ & $\begin{array}{l}\text { Unterstützung bspw. der Electronic Industry Citi- } \\
\text { zens Coalition (EICC) und Global e-Sustainability } \\
\text { Initiative (GeSI) }\end{array}$ & $\begin{array}{l}\text { Heydenreich und Schelhove } \\
(2010)\end{array}$ \\
\hline $\begin{array}{l}\text { Unterstützung von Initiativen und Organisatio- } \\
\text { nen, die Schulungsmaßnahmen für Minenarbeiter } \\
\text { forcieren }\end{array}$ & $\begin{array}{l}\text { Möglich; beispielsweise durch die Unterzeichnung } \\
\text { des von deutschen Nachhaltigkeitsrat initiierten } \\
\text { „Kodex für Nachhaltigkeit“ }\end{array}$ & Nachhaltigkeitsrat (2012) \\
\hline $\begin{array}{l}\text { Reduktion des Bedarfs an ökologisch kritischen } \\
\text { Rohstoffen }\end{array}$ & $\begin{array}{l}\text { Möglich, sofern dies auch techno-ökonomisch } \\
\text { möglich ist }\end{array}$ & $\begin{array}{l}\text { Ellis et al. (2007); BGR (2007, } \\
\text { S. 8); Angerer et al. (2009, S. 173) }\end{array}$ \\
\hline $\begin{array}{l}\text { Aktive Kommunikation des ökologischen } \\
\text { Engagements }\end{array}$ & Stets möglich & - \\
\hline
\end{tabular}

\section{Zusammenfassung und Ausblick}

Vor dem Hintergrund der starken Rohstoffabhängigkeit des produzierenden Gewerbes wurde im Rahmen dieses Beitrags dargelegt, dass sich ein Unternehmen bereits in der Entwicklungsphase eines Neuproduktes eingehend mit Rohstoffrisiken auseinandersetzen sollte. Im Rahmen dieses interdisziplinären Ansatzes wurden zunächst ökonomische, ökologische und soziale Fragestellungen erörtert, mit denen sich ein Unternehmen infolge seines Rohstoffbedarfs auseinandersetzen muss. Im Anschluss wurden mögliche Maßnahmen diskutiert, die zu einem nachhaltigeren Umgang mit knappen Rohstoffen beitragen können. Exemplarisch wurde hierzu näher auf das Metall Kobalt eingegangen, das beispielsweise bei der Herstellung von Hochleistungsakkumulatoren eine wichtige und kritische Rolle spielt.

Der Beitrag nimmt bewusst weder eine Priorisierung der drei Aspekte der Nachhaltigkeit noch eine Bewertung oder Reihung der dargestellten Maßnahmen vor. Dies liegt zum einen in der Tatsache begründet, dass eine Gewichtung jeweils subjektiv getroffen werden muss. Zum anderen sind diesbzgl. Entscheidungen unternehmensspezifisch und in Abhängigkeit von den jeweiligen Rohstoffbedarfen zu treffen. Mit diesem Vorgehen möchte der Beitrag einen breiten Leserkreis zu einer kritischen und aktiven Auseinandersetzung mit den Facetten nachhaltiger Neuproduktentwicklung anregen.

Im Rahmen weiterer Forschungsarbeiten bietet es sich einerseits an, die vorgestellten Maßnahmen auf ihrer positiven bzw. negativen Wechselwirkungen untereinander hin zu untersuchen. Zudem erscheint es sinnvoll, neben diesem auf das Neuproduktdesign fokussierten Beitrag auch wissenschaftliche Ansätze zu erarbeiten, welche Rolle politischen Entscheidungsträgern und Konsumenten bei der nachhaltigen Nutzung von Rohstoffen zukommt und wie sich diese miteinander verzahnen lassen.
Danksagung Der herzliche Dank des Autors gilt Prof. Dr. Andreas Rathgeber, Prof. Dr. Armin Reller und Prof. Dr. Axel Tuma sowie deren Mitarbeiterteams am Institut für Materials Resource Management (MRM) der Universität Augsburg, die die Entwicklung des Beitrags stets sehr konstruktiv unterstützt haben.

\section{Literatur}

Achzet B, Reller A, Zepf V (2011) Unternehmensstrategien zur Sicherung von Rohstoffen. Pusch Thema Umwelt 2:10-11

Angerer G, Erdmann L, Marscheider-Weidemann F, Scharp M, Lüllmann A, Handke V, Marwede M (2009) Rohstoffe für Zukunftstechnologien. Fraunhofer, Stuttgart

Benoît C, Mazijn B (2009) Guidelines for social life cycle assessment of products. Nairobi

Berger (2012) Global Vehicle LiB Market study update - global study. http://www.rolandberger.de/media/pdf/Roland_Berger_Li_Ion Batteries_Study_20120419.pdf. Zugegriffen: 27. Okt. 2012

BGR (2007) Rohstoffwirtschaftliche Steckbriefe für Metall- und Nichtmetallrohstoffe. Hannover

BGR (2010) „Coltan-Fingerprint“ der BGR macht Zertifizierung von Handelsketten möglich. http://www.bgr.bund.de/DE/Themen/ Min_rohstoffe/Projekte/Rohstoff-Forschung-abgeschlossen/LF_ Herkunftrsnachweis_COLTAN_Newsletter01-2010.html. Zugegriffen: 27. Okt. 2012

BGR (2011a) Deutschland Rohstoffsituation 2010. Hannover

BGR (2011b) Handbuch für die Zertifizierung der Erzes aus dem Zinnabbau in der DR Kongo. Kinshasa

BMU (2012) Nachhaltigkeit als Integrationsaufgabe. http://www.bmu. $\mathrm{de} /$ nachhaltige entwicklung/stategie und umsetzung/praktizierte_nachhaltigkeit/doc/2397.php. Zugegriffen: 27. Okt. 2012

BMZ (2010) Entwicklungspolitisches Strategiepapier Extraktive Rohstoffe. Bonn

Braess H-H, Seiffert U (Hrsg) (2005) Vieweg Handbuch Kraftfahrzeugtechnik, 4. Aufl. Vieweg, Wiesbaden

Buhl HU, Horn S, Reller A (2010) Den Engpass vermeiden. Automotive IT 1:32-33

Clausmark (2012) Rohstoffmanagement. http:/www.clausmark.com/ index.php/rohstoffmanagement.html. Zugegriffen: 27. Okt. 2012

Cobalt Development Institute (2012) Cobalt News April 2012. thecdi. com/cdi/images/news_pdf/cobalt_news_april12.pdfhttp://thecdi. com/cdi/images/news_pdf/cobalt_news_april12.pdf. Zugegriffen: 27. Okt. 2012 
DERA (2012) Rohstoffkooperationen. http://www.deutsche-rohstoffagentur.de/DERA/DE/Rohstofflaenderkooperationen/rohstofflaenderkooperationen_node.html. Zugegriffen: 27. Okt. 2012

Destatis (2007) Fachreihe produzierendes Gewerbe, verschiedene Jahrgänge; Fachreihe Volkswirtschaftliche Gesamtrechnung. http:// www.isi.fraunhofer.de/isi-de/n/download/publikationen/Schlussbericht lang 20090515 final.pdf. Zugegriffen: 27. Okt. 2012

Destatis (2012a) Inlandsproduktsberechnung - Bruttowertschöpfung nach Wirtschaftsbereichen. https://www.destatis.de/DE/ZahlenFakten/GesamtwirtschaftUmwelt/VGR/Inlandsprodukt/Tabellen/ BWSBereichen.htm. Zugegriffen: 27. Okt. 2012

Destatis (2012b) Bruttoinlands-Produkt 2011 für Deutschland. Wiesbaden

Deutsche Bank (2007) Rohstoff-Risiken managen. https:/www.globalbanking.db.com/docs/WiWo_Advertorial_Rohstoffe_0907. pdf. Zugegriffen: 27. Okt. 2012

Deutsche Bank (2012) Gefahren für das Geschäft - Umfrage unter Finanzentscheidern in 400 Unternehmen. Zitiert nach Handelsblatt 15. Juni 2012, S 42

DLR (2012) Interview mit DLR-Vorstand Prof. Ulrich Wagner. http:// www.dlr.de/dlr/presse/desktopdefault.aspx/tabid-10309/472 read-2862/year-all/. Zugegriffen: 27. Okt. 2012

EcoInvent (2010a) Datenbank Version 2.2, nach Frischknecht et al. (2007)

EcoInvent (2010b) Datenbank Version 2.2, nach IPCC (2007), Basis: 100 Jahre

Ellis BL, Makahnouk WRM, Makimura Y, Toghill K, Nazar LF (2007) A multifunctional 3.5 V iron-based phosphate cathode for rechargeable batteries. Nat Mater 6:749-753

EPI (2012) Environmental Performance Index. http://epi.yale.edu/ epi2012/rankings. Zugegriffen: 27. Okt. 2012

Erdmann L, Behrendt S, Feil M (2011) Kritische Rohstoffe für Deutschland. IZT, Berlin

EU (2010) Critical raw materials for the EU - report of the Ad-hoc Working Group on defining critical raw materials. Brüssel

EU (2011) Entschließung des Europäischen Parlaments vom 13. Sept. $2011 \mathrm{zu}$ einer erfolgreichen Rohstoffstrategie für Europa. Straßburg

EU (2012) Eine stärkere europäische Industrie bringt Wachstum und wirtschaftliche Erholung - Mitteilung zur Industriepolitik. http://eur-lex.europa.eu/LexUriServ/site/de/com/2012/ com2012 0582de01.pdf. Zugegriffen: 27. Okt. 2012

Faulstich M, Mocker M, Pfeifer S, Köglmeier M, Egner S (2010) r3 Innovative Technologien für Ressourceneffizienz - Strategische Metalle und Materialien. Straubing

Fraunhofer (2012) Pressemitteilung. http://www.isi.fraunhofer.de/ isi-de/service/presseinfos/2012/pri12-04.php. Zugegriffen: 27. Okt. 2012

Frondel M, Grosche P, Huchtemann D, Oberheitmann A, Peters J, Vance C, Angerer G, Sartorius C, Buchholz P, Rohling S, Wagner M (2007) Trends der Angebots- und Nachfragesituation bei mineralischen Rohstoffen. Essen

Goldman Sachs (2011) Commodity Price Volatility, 1991-present Goldman Sachs Commodity Index, 60-days. Zitiert nach Institut of International Finance (2011) Financial Investment in Commodity Markets: Potential Impact on Commodity Prices \& Volatility, S. 5. Washington

Gönner T (2010) Umwelttechnologie und Ressourceneffizienz in Baden-Württemberg. UmweltWirtschaftsForum 18(3-4): $181-187$

Graedel TE, Barr R, Chandler C, Chase T, Choi J, Christoffersen L, Friedlander E, Henly C, Jun C, Nassar NT, Schechner D, Warren S, Yang M, Zhu C (2011) Methodology of metal criticality determination. Environ Sci Technol 46:1063-1070

Handelsblatt (2012) Unternehmer gehen von höheren Rohstoffen aus. 21. Aug. 2012, S. 24
Heydenreich C, Schelhove C (2012) Ich sehe was, was Du nicht siehst - Die Schattenseiten der IT-Branche. Forum Nachhaltig Wirtschaften, 3

Hütz-Adams F (2012) Fact-Sheet: Die Wertschöpfungskette von Schokolade. Siegburg

IHK (2010) Rohstoffklemme zeichnet sich ab. http://www.muenchen. ihk.de/mike/ihk_geschaeftsfelder/starthilfe/Anhaenge/DIHKUnternehmensbarometer-Rohstoffe.pdf. Zugegriffen: 27. Okt. 2012

ILO (2012) International Labour Organization: Kernarbeitsnormen. http://www.ilo.org/public/german/region/eurpro/bonn/kernarbeitsnormen/index.htm. Zugegriffen: 27. Okt. 2012

Knoke I, Binnewies J (2010) Wem nutzt der Rohstoffreichtum? Siegburg

Krohns S, Lunkenheimer P, Meissner S, Reller A, Gleich B, Rathgeber A, Gaugler T, Buhl HU, Sinclair DC, Loidl A (2011) The route to resource-efficient novel materials. Nat Mater 10(12):899-901

LME (2010) LME launches cobalt and molybdenum futures trading. http://www.lme.com/media_resources/9862.asp. Zugegriffen: 27. Okt. 2012

LME (2012a) Warehouse rents. http://www.lme.com/downloads/ rentandfot/LME_Warehouse_rents_1_April_2012_to_31 March_2013C.xls. Zugegriffen: 27 . Okt. 2012

LME (2012b) LME cobalt and LME molybdenum futures trading features. http://www.lme.com/minormetals/6256.asp. Zugegriffen: 27. Okt. 2012

Metalprices (2012) Cobalt FOB Warehouse Rotterdam USD/KG. http://www.metalprices.com/metal/cobalt/cobalt-ingots-99-3-rotterdam. Zugegriffen: 27. Okt. 2012

Nachhaltigkeitsrat (2010) Chemischer „Fingerabdruck“ gegen illegalen Handel mit Rohstoff Coltan. http://www.nachhaltigkeitsrat. de/news-nachhaltigkeit/2010/2010-03-18/chemischer-fingerabdruck-gegen-illegalen-handel-mit-rohstoff-coltan/. Zugegriffen: 27. Okt. 2012

Nachhaltigkeitsrat (2012) Empfehlungen des Rates für Nachhaltige Entwicklung. Berlin

Oertel T (2003) Untersuchung und Bewertung geogener und anthropogener Bodenschwermetallanreicherungen als Basis einer geoökologischen Umweltanalyse im Raum Eisleben-Hettstedt. Halle

RA Rohostffalianz (2012) Rohstoffallianz - Unternehmenspräsentation. http://www.wbu-bayern.de/veranstaltungen/0920_Praesentation_Paskert.pdf. Zugegriffen: 27. Okt. 2012

Radetzki $\bar{M}$ (2008) A handbook of primary commodities in the global economy. Cambridge University Press, Cambridge

Reller A, Meißner S (2012) Vorlesung Nachhaltiges Ressourcenmanagement - Ressourcengeographie II. Augsburg

Slade ME (1982) Trends in natural-resource commodity prices: an analysis of the time domain. J Environ Econ Manage 9:122-137

Svedberg P, Tilton JE (2006) The real, real price of nonrenewable resources: copper 1870-2000. World Devel 34(3):501-519

Transparency International (2011) Corruption Perceptions Index 2011. http://www.transparency.de/Tabellarisches-Ranking.2021.0.html. Zugegriffen: 27. Okt. 2012

Treffer F (2011) Entwicklung eines realisierbaren Recyclingkonzeptes für die Hochleistungsbatterien zukünftiger Elektrofahrzeuge Lithium-Ionen Batterierecycling Initiative - LiBRi. Hanau

Tsurukawa N, Prakash S, Manhart A (2011) Social impacts of artisanal cobalt mining in Katanga, Democratic Republic of Congo. Freiburg

Tuseko BK (2001) RD Congo - Les Katangais accueillent à bras ouverts ceux qu'ils avaient chasses. http://www.syfia.info/index. php5 view $=$ articles\&action=voir\&idArticle=1261. Zugegriffen: 27. Okt. 2012 
US Embassy (2010) Recent allegations of uranium trafficking in the Democratic Republic of Congo. http://files.vpro.nl/wikileaks/ cable/2007/07/07KINSHASA797.html. Zugegriffen: 27. Okt. 2012

USGS (2010) Cobalt statistics and information. http://minerals.usgs. gov/minerals/pubs/commodity/cobalt/myb1-2010-cobal.xls. Zugegriffen: 27. Okt. 2012

USGS (2012) Mineral Commodity Summaries 2012. http://minerals. usgs.gov/minerals/pubs/mcs/2012/mcs2012.pdf. Zugegriffen: 27. Okt. 2012
Weltbank (2010) Wordwide Governance Indicators. http://info.worldbank.org/governance/wgi/worldmap.asp. Zugegriffen: 27. Okt. 2012

Yaksic A, Tilton JE (2009) Using the cumulative availability curve to assess the threat of mineral depletion: the case of lithium. Resources Pol 34(4):185-194 